\title{
Numerical simulation study on rheological failure characteristics of rock mass under high stress
}

\author{
Liming Qiu ${ }^{1,2, ~ *}$, Xueqiu $\mathrm{He}^{1,2}$, Dazhao Song ${ }^{1,2}$, and Zhenlei $\mathrm{Li}^{1,2}$ \\ ${ }^{1}$ School of Civil and Resource Engineering, University of Science and Technology Beijing, 100083 \\ Beijing, China \\ ${ }^{2}$ Key Laboratory of Ministry of Education for Efficient Mining and Safety of Metal Mine, University \\ of Science and Technology Beijing, 100083 Beijing, China
}

\begin{abstract}
This paper uses the RFPA numerical simulation software to establish a numerical model of the rheological failure of the rock mass under stress. Rheological failure characteristics of the body was researched, and the results shows: (1) The rupture sequence of rock rupture is from the corner to the middle. When the rock loses stability under pressure, the rock often ruptures from the corner. The corner gradually collapses and cracks. Then the cracks spread to the middle of the rock. Many cracks extending from the corners are in the rock. The central part intersects each other and eventually causes the rock to break. (2) Rock samples of different lithologies have different stress values when they break under the same confining pressure. From the experimental process, we know that granite>sandstone>mudstone. Therefore, the higher the strength of the rock, the harder the rock will be broken. (3) The weaker the plasticity at rupture, the stronger the brittleness and the stronger the sudden change of rupture. In the deep mining process, the greater the confining pressure, the more obvious the rheological characteristics of the rock, and the greater the total energy released during the rock failure process.
\end{abstract}

Keywords. deep mining, rock mass rheology, failure characteristics, numerical simulation.

\section{Introduction}

With my China's economic development and social progress, the demand for mineral resources has gradually increased, and the development technology of deep ground resources has continued to advance. The focus of mining and drilling engineering has gradually developed from shallow to deep. With the continuous deepening of mining depths, endless disasters have gradually come to people's eyes, such as high and low pressure, high water pressure, high gas and other issues. At the same time, taking into account the interaction between the mechanical characteristics of the mine, Resulting in a sharp increase in the probability and severity of disasters ${ }^{[1,2]}$.

\footnotetext{
* Corresponding author: qiulm@ustb.edu.cn
} 
The stability and rheological properties of deep high-stress coal has always been the focus and difficulty of mining workers. Scholars at home and abroad have conducted a lot of research on this problem. Heap et al. ${ }^{[3]}$ and Martin et al. ${ }^{[4]}$ conducted creep tests of sandstone, granite, basalt and other rocks under conventional triaxial compression conditions to study the failure events, velocity, compression deviator stress, surrounding rock and pore pressure of this phenomenon. The relationship between. Wang et al. ${ }^{[5]}$ studied the creep damage evolution characteristics of rock salt and gave the long-term strength characteristic curve of rock salt. Kang Hongpu ${ }^{[6]}$ and other scholars formulated the theory of high prestress and strong support for deep and difficult roadways. Wang Weijun [7] analyzed the formation and development process of plastic zone in high-stress soft rock roadway, and pointed out that the key to ensuring the stability of roadway surrounding rock is to control the local distortion of plastic ring and malignant expansion of plastic zone. Shen Mingrong et al. ${ }^{[8]}$ studied the shear creep characteristics of composite rock masses based on marble as the main body of the investigation, and proved that the specimens are more inclined to shear failure along the weak plane, and the characteristics of the rock mass also show three stages . However, there are few studies on the influence of stress on the failure strength of rock masses ${ }^{[9]}$. The rheological characteristics of rock masses under high stress are still unclear ${ }^{[10]}$. The prevention of rock mass deformation catastrophes during deep mining lacks sufficient theoretical guidance ${ }^{[11-13]}$.

Based on this, this paper intends to carry out a numerical simulation study on the rheological characteristics of rock mass under high stress, study the rheological properties of rocks, reveal the characteristics of rheological failure under high stress, and study the energy characteristics of rock deformation and failure under high stress ${ }^{[14]}$. Revealing the rheological failure conditions and precursor characteristics of rocks, and finally revealing the law and mechanism of the influence of high stress conditions on the failure of rock masses ${ }^{[15]}$. The above-mentioned researches play an important role in preventing disasters and safe production of mining, and have theoretical research on rock failure mechanisms. significance.

\section{Selection of numerical simulation parameters for rheological failure characteristics of rock mass}

In order to verify the failure characteristics of different lithological rocks, this paper selects three types of rocks such as mudstone, sandstone and granite for numerical simulation calculation. Numerical simulation experiments were carried out on the failure process of mudstone, sandstone and granite under the condition of confining pressure compression and their acoustic emission activities. The size of the rock model is determined according to $(50 \mathrm{~mm} \times 100 \mathrm{~mm}$ and $100 \mathrm{~mm} \times 100 \mathrm{~mm})$, The model will be divided into cell grids. Assuming that the mechanical parameters can be consistent with the Weibull statistical distribution, the damage criterion is selected as Mohr-Coulomb, the model is given seven sets of physical and mechanical parameters to simulate the deformation characteristics of granite, mudstone, sandstone and other samples under confining pressure compression. Refer to Table 1 below to understand the situation.

Table 1. Mechanical parameters of rock medium.

\begin{tabular}{llllllll}
\hline Lithology & Homogeneity & $\begin{array}{l}\text { Poisson's } \\
\text { ratio }\end{array}$ & $\begin{array}{l}\text { Density } \\
\left.\mathrm{kg} / \mathrm{m}^{3}\right)\end{array}$ & $\begin{array}{l}\text { Internal } \\
\text { friction } \\
\text { angle/ } \\
\left({ }^{\circ}\right)\end{array}$ & $\begin{array}{l}\text { Elastic } \\
\text { Modulus } \\
(\mathrm{MPa})\end{array}$ & $\begin{array}{l}\text { Rabbi } \\
\text { Compressive } \\
\text { strength } \\
\text { Mpa })\end{array}$ \\
\hline Mudstone & 3 & 0.26 & 1800 & 26.5 & 8000 & 16 & 30 \\
\hline
\end{tabular}




\section{Simulation research on rock confining pressure compression failure}

According to the model and parameter settings, the confining pressure compression simulation test of mudstone, sandstone and granite is continued with $5 \mathrm{MPa}, 15 \mathrm{MPa}$ and $25 \mathrm{MPa}$ respectively. The simulation results include fracture evolution process, acoustic emission analysis, etc. The following is a simulation of rock mass rheology under different confining pressure compression with side end openings. A numerical calculation model of $200 \mathrm{~mm} \times 200 \mathrm{~mm}$ is established. The number of grids is $100 \times 100$. The loading condition is confining pressure compression. Different, the single-step increment of stress varies from $0.5 \mathrm{MPa}$ to $4 \mathrm{MPa}$. The total loading step is controlled within 100 steps to simulate the deformation and fracture behavior under different stress conditions. The simplified plane model is a plane strain model. Through numerical simulation, observe the force characteristics and failure modes of different lithologies under different stresses, record the characteristics of acoustic emission and maximum shear stress, observe and analyze the failure modes of rocks under different stresses, observe the results and phenomena, and summarize high stress. The rheological law of rock mass under action.

\subsection{Rock failure modes under different stresses}

\section{(1) $5 \mathrm{MPa}$}

Under the condition of confining pressure of $5 \mathrm{MPa}$, the rupture evolution process of mudstone is shown in Figure 1. The top pressure and confining pressure remain unchanged at 5MPa, and the single-step increment is 0 . From the third loading step, the cavity excavation is carried out on the right side of the sample. It can be seen from Figure 1 that the length of mudstone remains unchanged, and the sample has no obvious cracks during the first loading step. From the third loading step, a cavity is excavated on the right side of the sample.

From the 3-1 loading step, micro-cracks began to appear at the two corners of the sample opening, and then the cracks gradually expanded. In the 3-5 loading step, the sample gradually collapsed inward from the two corners of the opening, the opening gradually deepened, the two corners near the opening changed from a right angle to an obtuse angle, and the sample gradually stabilized in the loading step 5-1.
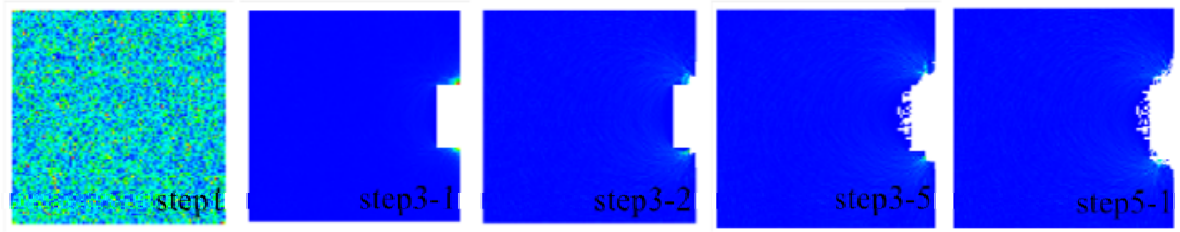

Fig. 1. Spatiotemporal evolution process of macroscopic failure induced by micro-element fracture of mudstone.
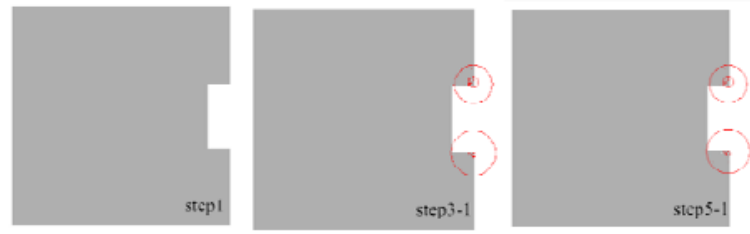

Fig. 2. Spatiotemporal evolution process of compressed acoustic emission in mudstone. 
It can be seen from Figure 2 that before the sample is opened, the sample does not deform, so there is no acoustic emission signal. Starting from the third loading step, the right side of the sample opens, and a small amount of acoustic emission energy is released at the two corners of the opening. And continue to the last loading step.

(2) $15 \mathrm{MPa}$

Under the condition of $15 \mathrm{MPa}$ confining pressure, the rupture evolution process of mudstone is shown in Figure 3 and Figure 4. The top pressure and confining pressure remain unchanged at $15 \mathrm{MPa}$, and the single-step increment is 0. From the third loading step, a cavity is made on the right side of the sample. Excavation. It can be seen from Figure 4 that the length of the mudstone remains unchanged, and the sample has no obvious cracks in the first loading step. From the third loading step, a cavity is excavated on the right side of the sample.

From the 3-1 loading step, micro-cracks began to appear at the two corners of the sample opening, and then the cracks gradually expanded. In the loading step 3-6, the sample gradually collapsed inward from the two corners of the opening, and the opening gradually deepened. The two corners near the opening changed from a right angle to an obtuse angle. In the loading step 3-10, the opening continued to deepen, and the upper right side of the sample began to face It collapses and gradually stabilizes at 10-1 loading step.

It can be seen from Figure 4 that before the sample is opened, the sample does not deform, so there is no acoustic emission signal. Starting from the third loading step, the right side of the sample opens, and a small amount of acoustic emission energy is released at the two corners of the opening. And continue to load step 10-1.
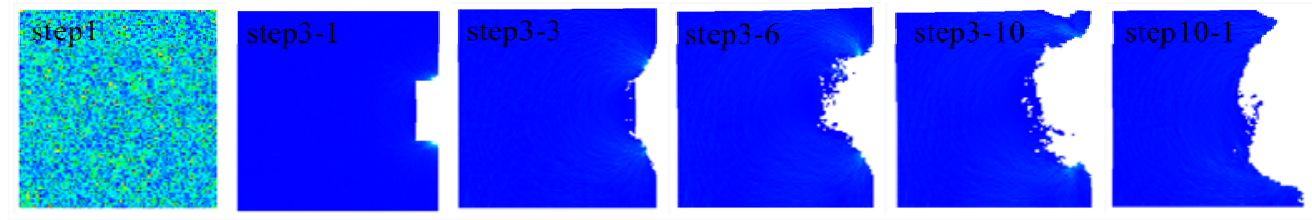

Fig. 3. Spatiotemporal evolution process of macroscopic failure induced by micro-element fracture of mudstone.
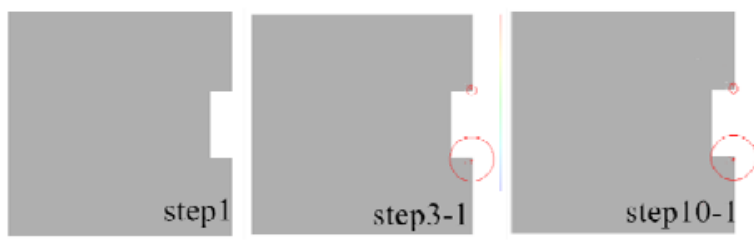

Fig. 4. Spatiotemporal evolution process of compressed acoustic emission in mudstone.

\section{(3) $25 \mathrm{MPa}$}

Under the condition of 25MPa confining pressure, the rupture evolution process of mudstone is shown in Figures 5 and 6. The top pressure and confining pressure remain unchanged at $25 \mathrm{MPa}$, and the single-step increment is 0 . Starting from the third loading step, a cavity is opened on the right side of the sample. dig. It can be seen from Figure 5 that the length of the mudstone remains unchanged, and the sample has no obvious cracks during the first loading step. From the third loading step, the cavity is excavated on the right side of the sample. Starting from the 3-1 loading step, the sample opens Micro-cracks began to appear in some of the two corners. In the 3-2 loading step, the cracks gradually expanded and the two corners quickly sag inward. In the loading steps 3-5, the sample collapses inward from the two corners of the opening, and the opening gradually deepens. The two corners near the opening change from a right angle to an obtuse angle. The 
opening continues to deepen in the loading steps 3-7, the upper right of the sample And the lower right opening began to collapse downward, and the sample was instability due to loading steps 3-10.

It can be seen from Figure 6 that before the sample is opened, the sample does not deform, so there is no acoustic emission signal. Starting from the third loading step, the right side of the sample opens, and a small amount of acoustic emission energy is released at the two corners of the opening. And continue to load step 3-10 sample instability.
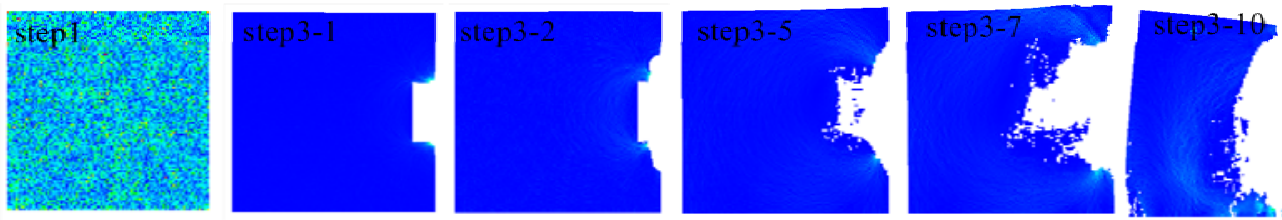

Fig. 5. Spatiotemporal evolution process of macroscopic failure induced by micro-element fracture of mudstone.
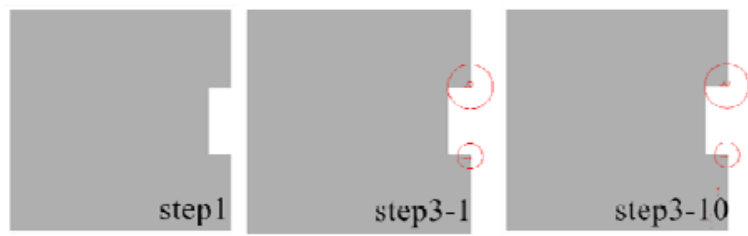

Fig. 6. Spatiotemporal evolution process of compressed acoustic emission in mudstone.

\subsection{Characteristics of rock failure energy under different stresses}

Since the acoustic emission energy and the acoustic emission count of the rock sample with the right side opening under confining pressure compression are the most representative of the opening, only the acoustic emission at the first loading step (step 3-2) after the opening is selected Energy and acoustic emission count data, and observe the energy characteristics of rock failure under the same confining pressure and different stress. It can be seen from Figure 7 that the energy change of the rock after the opening increases with the increase of the stress, and the effect of mudstone change is the most obvious: when the stress is 5MPa, the acoustic emission count of mudstone is 230 , at $15 \mathrm{MPa}$ The acoustic emission count is 2239 at $25 \mathrm{MPa}$, and the acoustic emission count of mudstone is 6646 at $25 \mathrm{MPa}$. This is because mudstone has a low compressive strength and therefore has a weaker compressive capacity. The deformation occurs when pressure is applied to the gap. Larger, the released energy is also greater, and the acoustic emission energy is released at 25MPa. The reason is that after opening at $25 \mathrm{MPa}$, the sample gradually becomes unstable, so a large amount of energy is released.

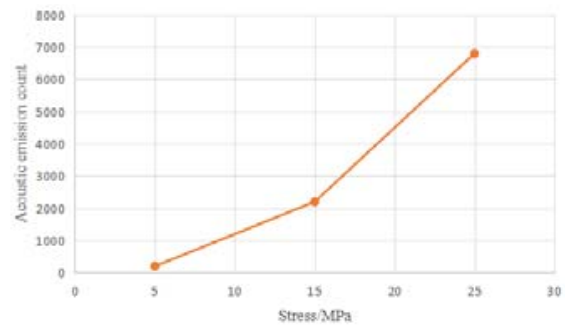

Fig. 7. Mudstone stress-acoustic emission counting curve. 


\section{Conclusion}

This paper numerically simulates the failure process of sandstone, mudstone and granite under different stresses, analyzes the influence of side end openings on the failure characteristics of rock under different stress conditions and the failure energy characteristics of different stresses and lithologies. The main conclusions are as follows:

(1) The rock gradually breaks with the appearance of side openings under a fixed confining pressure. The failure process starts from around the opening, and the four corners break faster than the middle, and gradually break into the sample; according to the rock breaking process The rupture sequence of rock fracture is the corners and then the middle. If the rock loses stability under pressure, it often starts to rupture from the corners, the corners gradually collapse and fracture, and then the cracks spread to the middle of the rock. Many cracks extending from the corners are in the middle of the rock. They cross each other and cause the opening to become larger and larger from the right side to the middle, eventually causing the rock to break;

(2) Under different lateral stresses, the rock initiation stress and instability strength are different. With the increase of lateral stress, the rock initiation stress and instability strength gradually increase, and mudstone occurs at 25MPa Obvious instability and neither sandstone nor granite is instability, and the instability effect of granite is less obvious than that of sandstone;

(3) Different rocks have different failure characteristics of side openings under the same confining pressure. Under the same confining pressure, mudstone has more obvious fracture characteristics than sandstone granite, and its failure strength is greater. When damaged, the acoustic emission energy is released from the two corners of the opening, and the sample gradually collapses from the two corners of the opening, the opening gradually deepens, and it ruptures from the corner to the middle. In the deep mining process, the greater the confining pressure, the more obvious the rheological characteristics of the rock, and the greater the total energy released during the rock failure process.

\section{Acknowledgements}

This work was financially supported by the Fundamental Research Funds for the Central Universities (No. FRF-TP-18-081A1), the Postdoctoral Science Foundation of China (No. 2018M641201), and the National Natural Science Foundation of China (Nos. 51634001, 51904019).

\section{References}

1. Yu Guohua, Wu Jianjia, Private Technology, 11, 111-113 (2017)

2. Xie Heping, Gao Feng, Ju Yang, Chinese Journal of Rock Mechanics and Engineering, 34(11), 2161-2178 (2015)

3. M.J. Heap, P. Baud, P.G. Meredith, S. Vinciguerra, A.F. Bell, I.G. Main, Earth and Planetary Science Letters, 307(1), 71-82 (2011)

4. R.J. Martin, J.S. Noel, P.J. Boyd, R.H. Price, International Journal of RockMechanics and Mining Sciences, 34(3-4), 190-199 (1997)

5. G.J. Wang, L. Zhang, Y.W. Zhang, International Journal of Rock Mechanics and Mining Sciences, 66(1), 181-187 (2014)

6. Sun Xiaoming, He Manchao, Journal of China University of Mining and Technology, 2, 37-40 (2005)

7. Yu Jianwei, Wang Weijun, Huang Wenzhong, Wu Hai, Journal of China Coal Society, 39(4), 614-623 (2014) 
8. Sheng Mingrong, Zhang Qingzhao, Chinese Journal of Rock Mechanics and Engineering, 29(6), 1149-1155 (2010)

9. Zhou Hongwei, Xie Heping, Zuo Jianping, Advances in Mechanics, 35(1), 91-99 (2005)

10. M. Kwasniewski, Laws of Brittle Failxire And of B-D Transition In Sandstones (1989)

11. M S. Paterson, Geological Society of America Bulletin, 69(4), 465 (1958)

12. D. F. Malan, Rock Mechanics and Rock Engineering, 535(4), 225-254 (2002)

13. Q Gong, L Yin, S Wu, et al, Engineering Geology, 124(1), 98-108 (2012)

14. Lei Shaohua, Zhang Lin, Ma Zhenkai, Shaanxi Coal, 36(6), 64-66 (2017)

15. He Manchao, Qian Qihu. Foundation of Deep Rock Mass Mechanics (Beijing: Science Press, 2010) 